\section{OP0136 SHOULD WE ERADICATE HELICOBACTER PYLORY BEFORE PRESCRIBING AN NSAID ? RESULT OF A PLACEBO CONTROLLED STUDY}

${ }^{1} \mathrm{~T}$ Schaeverbeke, ${ }^{2} \mathrm{~N}$ Broutet, ${ }^{3} \mathrm{~F}$ Zerbib, ${ }^{4} \mathrm{~B}$ Combe, ${ }^{5} \mathrm{P}$ Bertin, ${ }^{6} \mathrm{~F}$ Perié, ${ }^{6} \mathrm{M}$ Joubert-Collin, ${ }^{2} \mathrm{~F}$ Mégraud. 'Rheumatology; ${ }^{2}$ Bacteriology; ${ }^{3}$ Hepato-Gastroenterology, CHU de Bordeaux, Bordeaux; ${ }^{4}$ Rheumatology, CHU de Montpellier, Montpellier; ${ }^{5}$ Rheumatology, CHU de Limoges, Limoges; ${ }^{6}$ Laboratoires, Takeda, Puteaux, France

10.1136/annrheumdis-2001.861

Background The influence of the infection by Helicobacter pylori $(\mathrm{Hp})$ on the gastro-intestinal (GI) tolerability of NSAIDs remains unknown.

Objectives 1) to determine the prevalence of GI symptoms in patients treated with NSAIDs as they are or not infected by $\mathrm{Hp}$, 2) to estimate the impact of $\mathrm{Hp}$ eradication on the prevalence of these symptoms.

Methods Multicentric, randomised, double blind, placebo-controlled study. Selection: patients presenting with a degenerative articular disease motivating the prescription of an NSAID for a duration $>=2$ weeks. Distribution of the patients in two groups $\mathrm{Hp}$-positive and $\mathrm{Hp}$-negative by a serologic fast digital test. Randomisation of $\mathrm{Hp}$-positive patients in two subgroups, receiving an eradication treatment: lansoprazole $60 \mathrm{mg} / \mathrm{d}$, amoxicilline $2 \mathrm{~g} / \mathrm{d}$ and clarithromycine $1 \mathrm{~g} / \mathrm{d}$ during 7 days (group 1), or a placebo (group 2). Prevalence of GI symptoms estimated in the 3 groups (group $3=$ not infected patients) at 2, 6 and 12 weeks after the beginning of the treatment. Analysis in intention to treat.

Results 330 patients; group 1: $\mathrm{n}=93$; group 2: $\mathrm{n}=92$; group $3: \mathrm{n}=145$. The highest prevalence of GI symptoms in the group 1 at $\mathrm{S} 2$ was related to diarrhoea, main side effect of antibiotics $(21,8 \%$ vs $4,6 \%$ in the group 2 and $5,6 \%$ in the group $3)$. The prevalence of GI symptoms at S6 was not different between the groups 2 and $3(\mathrm{p}=0,77)$. After adjustment of the NSAIDs dosage received between S6 and S12, the prevalence of GI symptoms was the same between group 1 and e group 3 $(9.4 \%$ and $10.0 \%)$. This prevalence was lower than in group 2 (17.3\%). This difference was not significant because of the samples size, but the most frequent GI symptom identified in this group was pyrosis.

\begin{tabular}{llll}
\multicolumn{4}{l}{ Abstract OP0136 Table 1 } \\
\hline Group 1 & Group 2 & Group 3 \\
\hline S2 & $57,7 \%$ & $40,7 \%$ & $42,6 \%$ \\
S6 & $24,7 \%$ & $23 \%$ & $21,4 \%$ \\
S12 & $9,4 \%$ & $17,3 \%$ & $10,0 \%$ \\
\hline
\end{tabular}

Conclusion The GI tolerance of conventional NSAIDs does not differ whether or not patients are infected by $\mathrm{Hp}$. Systematic $\mathrm{Hp}$ eradication in infected patients does not seem to improve the digestive tolerance of NSAIDs for the short-term; medium-term observed tendency deserves to be confirmed.

\section{OP0137 LESS HEALTHCARE RESOURCE UTILISATION FOR PERFORATIONS, ULCERS AND BLEEDS WITH ROFECOXIB VS. NAPROXEN IN THE VIGOUR (VIOXX GI OUTCOMES RESEARCH) STUDY}

${ }^{1} \mathrm{C}$ Bombardier, ${ }^{2} \mathrm{~L}$ Laine, ${ }^{3} \mathrm{GW}$ Carides, ${ }^{3} \mathrm{JM}$ Pellissier, ${ }^{3} \mathrm{DR}$ Ramey, ${ }^{3} \mathrm{DJ}$ Watson, ${ }^{3} \mathrm{~A}$ Reicin. ${ }^{1}$ Clinical Epidemiology Division, Wellesley Hospital Research Institute, Toronto, Canada; ${ }^{2}$ USC School of Medicine, Los Angeles, CA, USA; ${ }^{3}$ Merck Research Laboratories, West Point, $P A, U S A$

\subsection{6/annrheumdis-2001.862}

Background The VIGOUR Study was a randomised, doubleblind, clinical trial assessing the GI safety of rofecoxib $50 \mathrm{mg}$. once daily $(\mathrm{n}=4047)$ versus naproxen $500 \mathrm{mg}$ twice daily $(\mathrm{n}=$ 4029) in the patients with rheumatoid arthritis. The mean age was 58 years; $80 \%$ of patients were female; $8 \%$ had a prior history of a GI ulcer or bleed; $82 \%$ reported NSAID use at baseline; $21 \%$ reported dyspepsia within 14 days prior to baseline; the treatment groups were not different in these respects. The median duration of follow-up 9.0 months, the maximum was 13 months.

Objectives To compare the rates of hospitalisation and mean number of outpatient healthcare contacts associated with upper GI perforation, ulcer, or bleeding (PUB) in patients treated with rofecoxib, a highly selective COX-2 inhibitor, vs. naproxen in the VIGOUR Study.

Methods The proportions of patients with hospitalizations for PUB in the two treatment groups were compared for all randomised patients. All PUBs were confirmed by an outside adjudication committee. The proportions of patients hospitalised for PUB, and the rates of PUB hospitalisation (per 100 patient-years, and based on the Kaplan-Meier method) were compared between treatment groups. Additional analyses compare proportions of PUB patients hospitalised and the mean number of outpatient contacts given a PUB event.

Results The incidence of confirmed PUBs, the primary endpoint in VIGOUR, was significantly lower with rofecoxib compared to naproxen ( 2.1 vs $4.5 \%$ per year; $\mathrm{p}<0.001$, relative risk 0.46 ). Patients treated with rofecoxib were hospitalised for PUB less often than patients treated with naproxen (crude rate: $0.4 \%$ vs $0.8 \%, 95 \%$ CI of difference $-0.7 \%$ to $-0.1 \%)$. Fewer patients treated with rofecoxib versus naproxen were hospitalised for complicated PUBs (i.e., perforation, obstruction, or clinically serious bleeding) $(0.2 \%$ vs $0.6 \%, 95 \% \mathrm{CI}$ of difference- $0.6 \%$ to $-0.1 \%)$; there was no difference between groups in the proportion of patients hospitalised with uncomplicated PUBs $(0.2 \%$ both groups). PUB hospitalisation rates per 100 patient years were lower rofecoxib than for naproxen $(0.63$ vs. 1.26 , risk ratio $0.50,95 \%$ CI of risk ratio 0.28 to 0.89 ). These rates were higher in the US for both treatment groups (rofecoxib 0.91, naproxen 1.65) compared with ex-US (rofecoxib 0.44, naproxen 1.00), however the reduction in rates with rofecoxib was similar in the US and ex-US. Analyses of the one-year hospitalisation incidence rates for PUBs based on the Kaplan-Meier method produced analogous results. As expected, in those patients who experienced a PUB, rates of outpatient contacts and hospitalizations were not different between treatment groups.

Conclusion In the VIGOUR Study, RA patients treated with the highly selective COX-2 inhibitor rofecoxib for up to 13 months had significantly fewer PUBs and required fewer hospitalizations for PUBs than patients treated with naproxen. 